\title{
Subcutaneous Implantation of Poly(3-hydroxybutyrate-co-3-hydroxyvalerate) and Poly(E-caprolactone) Scaffolds Modified with Growth Factors
}

\author{
DOI: $10.17691 / \mathrm{stm} 2017.9 .2 .01$
}

Received November 11, 2016

\begin{abstract}
M.V. Nasonova, Researcher, Laboratory of Novel Biomaterials, Division of Experimental and Clinical Cardiology;

D.K. Shishkova, Junior Researcher, Laboratory of Novel Biomaterials, Division of Experimental and Clinical Cardiology;

L.V. Antonova, MD, PhD, Head of the Laboratory of Cell Technologies, Division of Experimental and Clinical Cardiology;

V.V. Sevostyanova, MD, PhD, Researcher, Laboratory of Novel Biomaterials, Division of Experimental and Clinical Cardiology;

Y.A. Kudryavtseva, DSc, Head of Experimental and Clinical Cardiology Division;

O.L. Barbarash, MD, DSc, Professor, Corresponding Member of the Russian Academy of Sciences, Director;

L.S. Barbarash, MD, DSc, Professor, Academician of the Russian Academy of Sciences, Chief Researcher

Research Institute for Complex Issues of Cardiovascular Diseases, 6 Sosnoviy blvd, Kemerovo, 650002, Russian Federation
\end{abstract}

The aim of the investigation was to assess the tissue response to subcutaneous implantation of nonwoven scaffolds fabricated from biodegradable polymers and further modified by growth factors.

Materials and Methods. Specimens were produced by two-phase electrospinning from poly(E-caprolactone) and a blend of poly(3hydroxybutyrate-co-3-hydroxyvalerate) and poly( $\varepsilon$-caprolactone) both unmodified (controls) and modified with growth factors VEGF, bFGF, and SDF-1 $\alpha$. The ratio of a polymer solution in chloroform and a water phase with a bioactive molecule was 20:1. To study the tissue response in vivo, nonwoven scaffolds were subcutaneously implanted in Wistar rats weighing $80-100 \mathrm{~g}$ for the periods of either 1, 2, 3, 6, 9 , or 12 months.

Results. Incorporating growth factors into the scaffolds during electrospinning provided biofunctionalization, including enhanced vasculogenesis and angiogenesis and increased viability of endothelial cells. Histological examination showed that biomolecules incorporated into the matrix have been functionally active throughout the whole time of the implantation.

The tested specimens did not cause rejection and acute inflammatory reaction. A thin connective tissue capsule was formed around the implants. A full resorption of the scaffolds did not occur, and the polymers remained at the site of implantation for at least 12 months. Growth factors significantly improved performance of the implants during the first 3 months postimplantation: VEGF enhanced angiogenesis, bFGF stimulated a thick connective tissue capsule formation, while SDF-1a facilitated angiogenesis and cellular infiltration. From the $12^{\text {th }}$ month postimplantation, incomplete biodegradation of nonwoven scaffolds caused granulomatous inflammation.

Conclusion. Nonwoven scaffolds fabricated of biodegradable polymers and further modified with VEGF, bFGF, and SDF-1a represent a promising option for the fabrication of cardiovascular implants.

Key words: scaffolds; biodegradable polymers; growth factors; nonwoven scaffolds; VEGF; bFGF; SDF-1a; poly(E-caprolactone); poly(3hydroxybutyrate-co-3-hydroxyvalerate); electrospinning.

For contacts: Marina V. Nasonova, e-mail: mv-nas@mail.ru 
Creation of electrospun three-dimensional fibrous structures (nonwoven scaffolds) having a high surfaceto-volume ratio and imitating extracellular matrix are of particular interest for regenerative surgery [1-4]. These constructs provide a structural basis and support optimal conditions of metabolism and cell differentiation, promoting vascularization and tissue remodeling. This approach may be successfully utilized in vascular tissue engineering, particularly for the fabrication of smalldiameter grafts. Poly( $\varepsilon$-caprolactone) (PCL) and poly(3hydroxybutyrate-co-3-hydroxyvalerate (PHBV) possess optimal biomechanical properties and biodegradation rate for the production of vascular grafts [5-8].

To increase bio- and hemocompatibility of vascular grafts, they can be seeded with endothelial, smooth muscle cells, or fibroblasts [1,9]. However, there are technical difficulties concerning isolation of these cells and their low proliferating activity that increases the time for obtaining a considerable number of cells [4]. Furthermore, application of autologous cells is also limited by their low viability in aged patients with cardiovascular disease [10]

An alternative to this technology is the application of bioactive molecules to stimulate de novo tissue formation. The most promising approach seems to be a creation of a bioactive medium for regeneration of blood vessels on the polymer scaffolds without any cell seeding [9]. Growth factors are widely used for this purpose as they are able to regulate cell migration, proliferation, differentiation, and apoptosis [11]. Incorporation of the growth factors during electrospinning makes possible to endow the scaffolds with biofunctional properties in order to regenerate vascular tissues.

Vascular endothelial growth factor (VEGF) is the most applied molecule for stimulation of graft endothelialization, as it is the most important regulator of vessel development in embryogenesis (vasculogenesis) and vascular formation in an adult organism (angiogenesis) [12]. Basic fibroblast growth factor (bFGF) is a mitogen for fibroblasts and plays a significant role in tissue remodeling and regeneration. bFGF stimulates quiescent endothelial cells, inducing their proliferation and organization into the tubular structures [13]. Stromal cell-derived factor-1a (SDF$1 \alpha$ ) is of great interest as a regulator of blood vessel formation, and though it is not referred to growth factors, it possesses a marked angiogenic activity.

Development of cardiovascular implants requires performance of in vivo implantation in order to evaluate local harmful effects in a living organism [14]. Implantation following explantation of the grafts with adjacent tissues provides the opportunity to assess the organism response.

The aim of this investigation was to evaluate the response of the surrounding tissues to $\mathrm{PCL}$ and $\mathrm{PHBV} /$ PCL scaffolds modified with VEGF, bFGF, and SDF-1a using a rat subcutaneous implantation model.

Materials and Methods. Nonwoven scaffolds from
$\mathrm{PCL}$ and PHBV/PCL, either nonmodified (controls) or modified with VEGF, bFGF, and SDF-1a, were fabricated by two-phase electrospinning (NANON-01A; MECC Co., Ltd., Japan). Electrospinning parameters were as follows: a ratio of polymer/chloroform solution to phosphate buffered saline/bioactive molecule 20:1; voltage of $20 \mathrm{kV}$; feed rate of $0.3 \mathrm{~mL} / \mathrm{h}$; $200 \mathrm{rpm}$ collector rotation speed; tip-to-collector distance of $15 \mathrm{~cm}$; $27 \mathrm{G}$ needle. For the fabrication of PHBV/PCL grafts the voltage was $23 \mathrm{kV}$, with other parameters similar to those used for PCL grafts.

To study the tissue response in vivo, nonwoven scaffolds were subcutaneously implanted in Wistar rats weighing $80-100 \mathrm{~g}$ for the periods of either $1,2,3,6,9$, or 12 months.

Immediately before implantation, grafts were sterilized with ethylene oxide at room temperature. Implantation was performed in a standard way [15]. Once the experiment was accomplished, scaffolds were explanted along with the adjacent tissues, fixed in $4 \%$ neutral phosphate-buffered formalin, and embedded in paraffin. Sections were stained with hematoxylin and eosin and van Gieson stain following light microscopy examination (Axio Imager A1; Carl Zeiss, Germany) with $\times 100$ magnification.

The work was performed in accordance with the European Convention for the Protection of Vertebrate Animals used for Experimental and other Scientific Purposes (the Convention was passed in Strasbourg, March 18, 1986, adopted in Strasbourg, June 15, 2006) and was approved by Ethical Committee of the Research Institute for Complex Issues of Cardiovascular Diseases.

We evaluated the following parameters: connective tissue capsule thickness, the number of de novo formed vessels in the scaffold and adjacent tissues, cellular infiltration in five vision fields, and polymer resorption rate.

Results and Discussion. 1 month postimplantation there were no signs of scaffold degradation in all specimen groups (Figure 1). Specimens were enclosed in the capsule from a loose connective tissue containing a minor amount of extracellular matrix and numerous cells, mainly lymphocytes, macrophages and fibroblasts. An intensive macrophagal and fibroblastic reaction were noted on the graft-tissue border. In the reactive zone around the scaffolds, de novo formed blood vessels were detected. The highest number of vessels was observed in the scaffolds with VEGF in comparison with all tested groups (Figure 1 (c), (d)). In the group of $\mathrm{PHBV} / \mathrm{PCL}$ scaffolds with bFGF, a significant number of sequentially located thin-wall blood vessels were observed along the scaffold (Figure $1(\mathrm{f})$ ). The specimens of this group were surrounded by the thick, loose, multilayer connective tissue capsule.

At the same time, single de novo formed blood vessels were found inside the scaffolds with incorporated SDF$1 \alpha$, and intensive infiltration of the scaffold by fibroblasts and macrophages was observed (Figure $1(\mathrm{~g}),(\mathrm{h})$ ). 

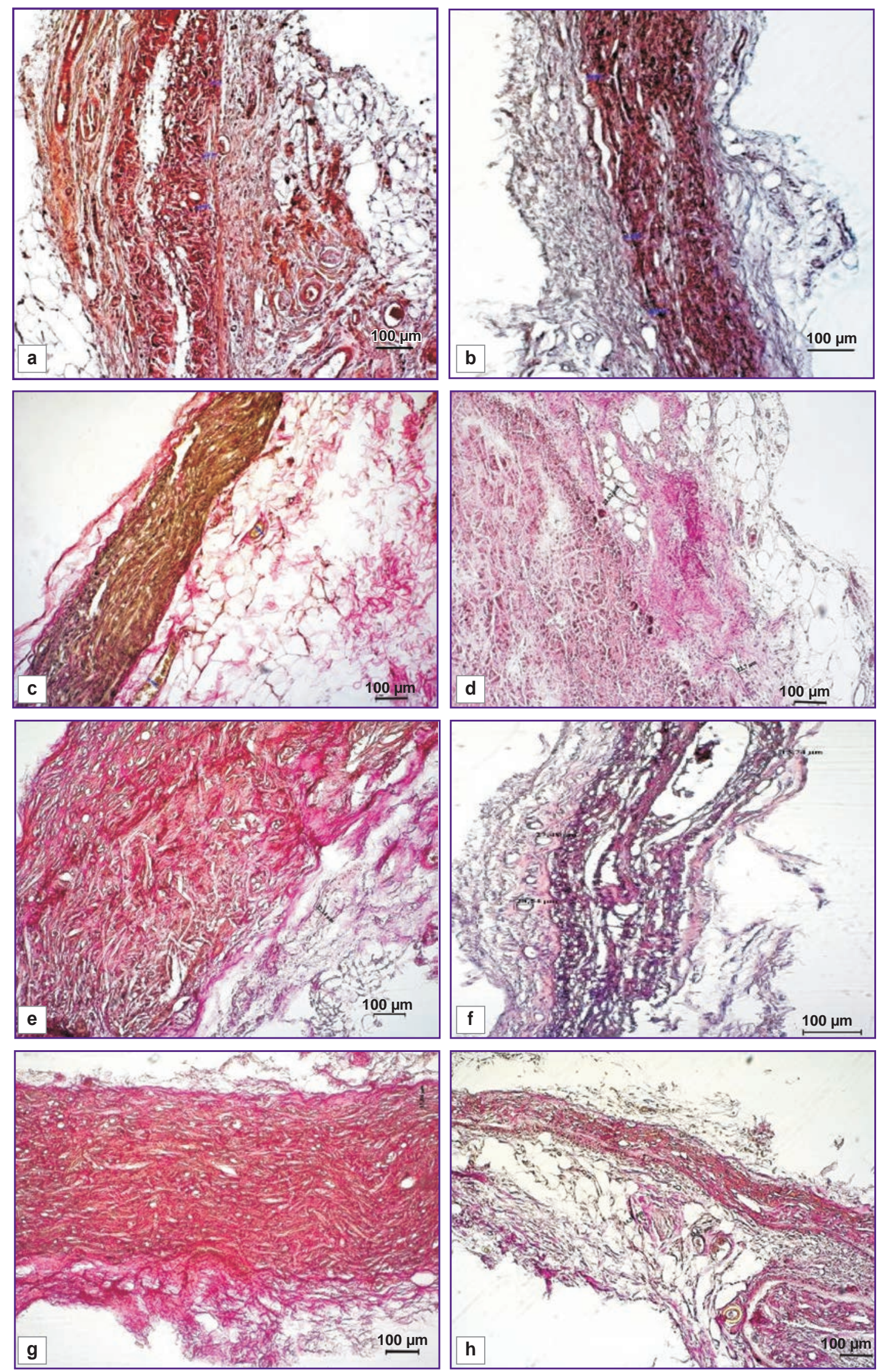

Figure 1. Nonwoven scaffolds 1 month postimplantation, $\times 100$ :

(a) PCL; (b) PHBV/PCL; (c) PCL+VEGF; (d) PHBV/PCL+VEGF; (e) PCL+bFGF; (f) PHBV/PCL+bFGF;

(g) PCL+SDF-1a; (h) PHBV/PCL+SDF-1a 

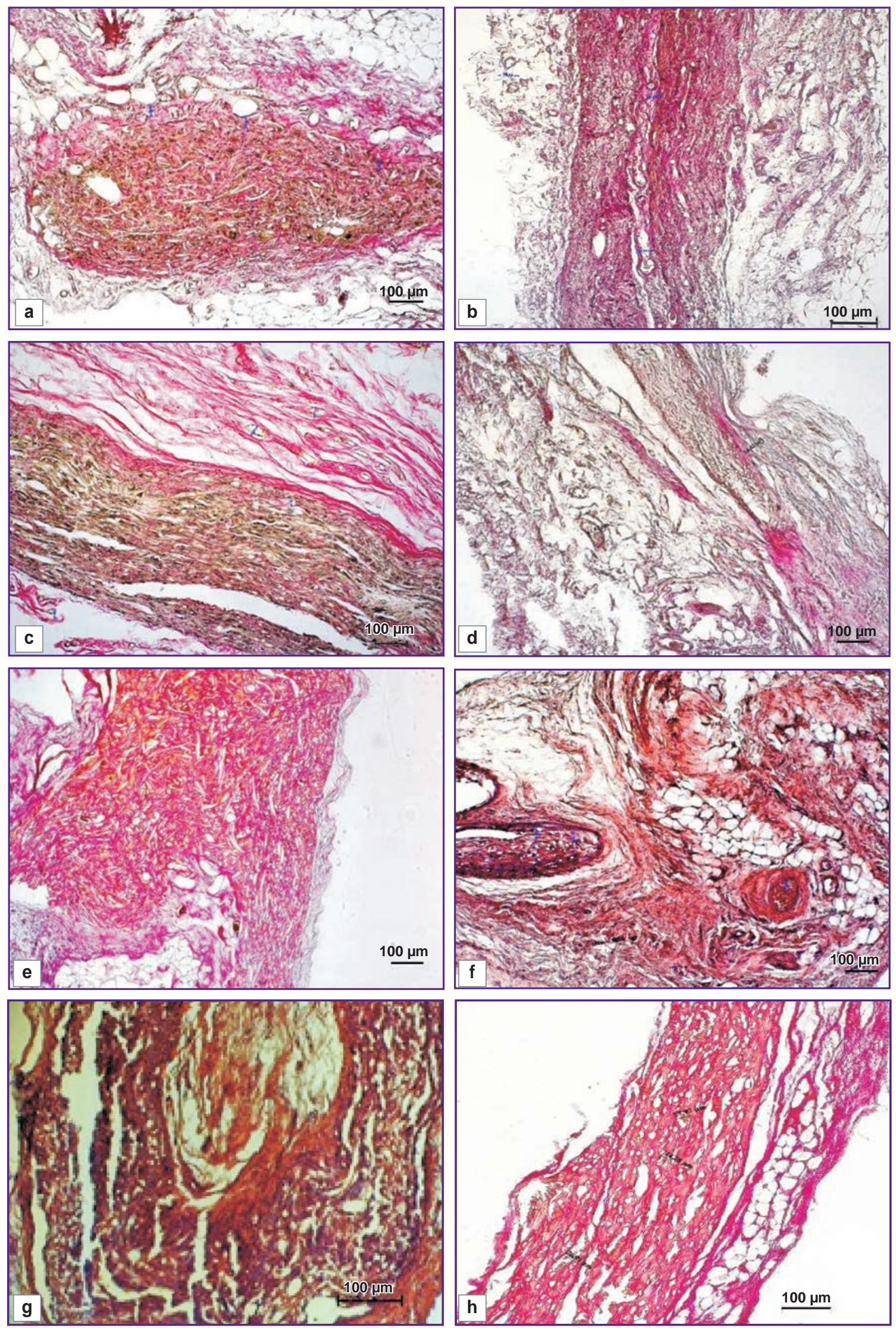

Figure 2. Nonwoven scaffolds 2 months postimplantation, $\times 100$ :

(a) PCL; (b) PHBV/PCL; (c) PCL+VEGF; (d) PHBV/PCL+VEGF; (e) PCL+bFGF; (f) PHBV/PCL+bFGF;

(g) PCL+SDF-1 $\alpha$; (h) PHBV/PCL+SDF-1 $\alpha$ 

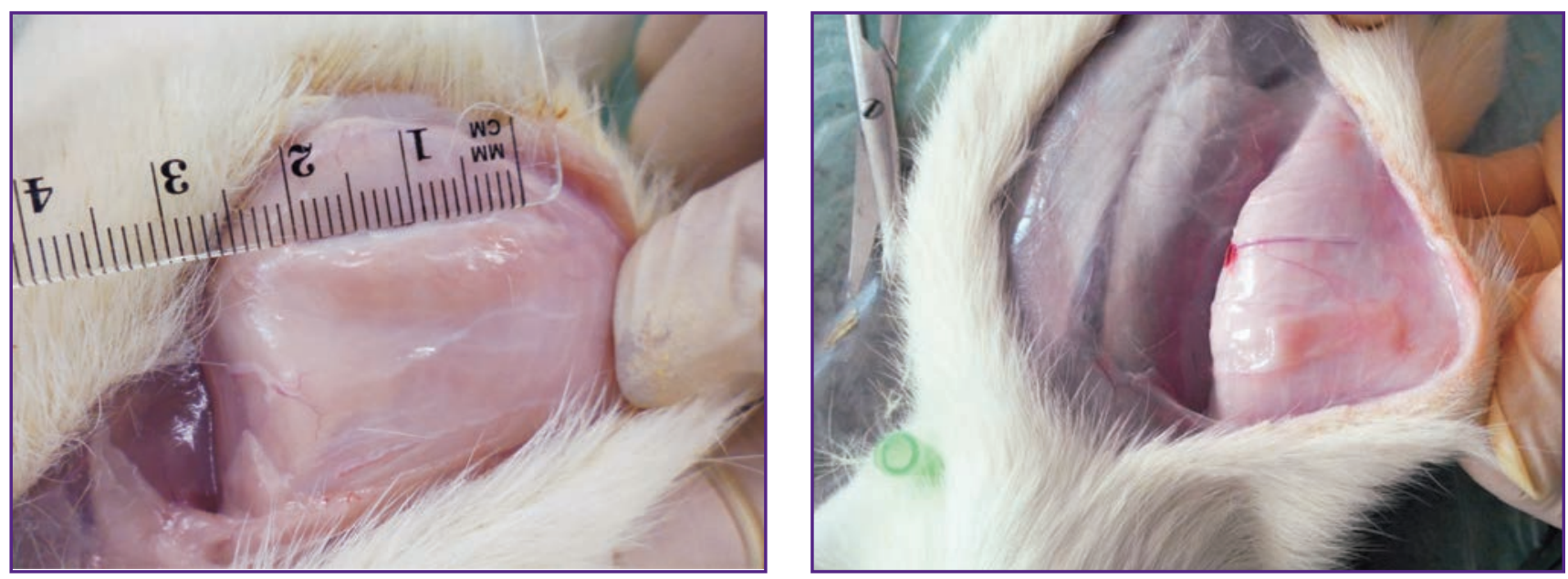

Figure 3. Nonwoven scaffolds from PCL 3 months postimplantation

2 months postimplantation, there were no significant differences in the tissue conditions and in the structure of the fibrous capsule around the scaffold. A high number of macrophages have been still visualized, being the evidence of intensive matrix resorption. Polymer specimens were divided into oval fragments surrounded by a thin, dense connective tissue capsule with a weak leukocytic infiltration (Figure 2).

3 months postimplantation, PCL scaffolds with and without growth factors underwent 2.8-fold stretching in longitudinal direction (Figure 3). It probably was due to a higher elasticity of PCL compared to PHBV/ PCL scaffolds. Since the specimens were implanted on the dorsal side of the animal, rat movements could exert a mechanical load on the elastic material causing a stretch. This effect could be also due to the fact that the specimens were implanted to the young animals weighing 80-100 g, which were actively developing and enlarging in size after implantation, and scaffolds extended due to their high elasticity as the animals grew. When similar scaffolds were implanted on the anterior surface of the heart in the previous experiments, this effect was not observed [5].

A weak leukocytic infiltration of connective tissue capsule was noted in all types of specimens. 3 months postimplantation the capsule was characterized by a strongly marked bilayer structure (Figure 4). The inner layer occupied $1 / 3$ of the thickness and was represented by a loose fibrous tissue with a large quantity of macrophages, fibroblasts and giant cells of foreign bodies. The outer layer was represented a dense fibrous tissue in the form of bundles of collagen fibers and fibrocytes adjoining to them, with a depletion of the infiltrate cellular composition. In the scaffolds with VEGF, the greatest number of vessels were observed relative to other groups (Figure 4 (c), (d)). In the group of scaffolds with incorporated bFGF, intensive fibroblastic infiltration was noted (Figure 4 (e), (f)). An extracellular matrix was located around the fibroblasts, and the number of cells populating the scaffolds essentially increased by the third month of the experiment. Connective tissue capsule around the specimens of this group had a greater thickness compared to the controls and scaffolds with VEGF and SDF-1 1 . In the scaffolds with SDF-1a, single vessels in the fields of vision were visualized (Figure 4 (g), (h)).

6 months postimplantation, degenerative processes were clearly seen at the outer matrix surface. Dissociation of the biomaterial and its lymphohistiocytic infiltration took place. A newly formed collagen fibers were noted not only around the transplant but in the matrix depth as well. This process was the most intensive in the scaffolds with SDF-1a (Figure $5(\mathrm{~g})$, (h)). In the group of specimens with incorporated VEGF, a decrease of the vessel quantity was noted (Figure 5 (c), (d)), in the scaffolds with bFGF, bundles of connective tissue were visualized (Figure 5 (e), (f)).

9 months postimplantation, a further destruction of the biomaterial with the scaffold dissociation was going on. In scaffolds with bFGF, foci of fibrosis were observed (Figure 6 (e), (f)), in those with VEGF there was a weak lymphocytic infiltration (Figure 6 (b), (c)). In all groups of experimental specimens, single vessels in the fields of vision were visualized. It is necessary to note that replacement of PHBV/PCL scaffolds with a connective tissue occurred by layers with preservation of the framework of the matrix itself (Figure 6 (b), (d)), in the specimens with a pure PCL, the tissue growth was chaotic (Figure 6 (a), (c)).

12 months postimplantation, the histological picture looked as follows: granulomatous inflammation continued, a weak matrix vascularisation was noted in all tested groups, and replacement of fibroblasts by fibrocytes was going on (Figure 7). In the depth of the scaffolds with incorporated VEGF, connective tissue interlayers were noticed (Figure 7 (c), (d)). 

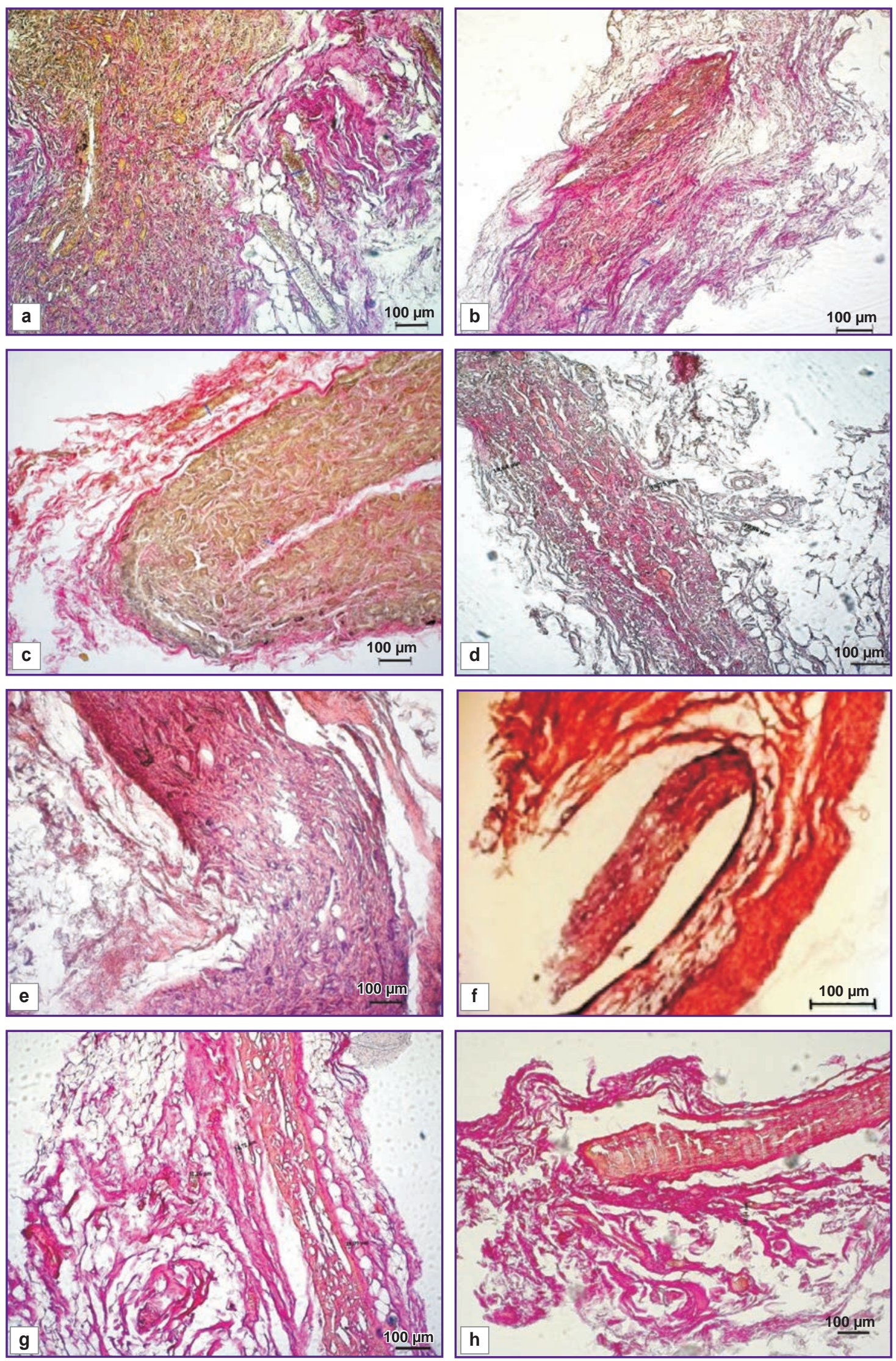

Figure 4. Nonwoven scaffolds 3 months postimplantation, $\times 100$ :

(a) PCL; (b) PHBV/PCL; (c) PCL+VEGF; (d) PHBV/PCL+VEGF; (e) PCL+bFGF; (f) PHBV/PCL+bFGF;

(g) PCL+SDF-1 $\alpha$; (h) PHBV/PCL+SDF-1 $\alpha$ 

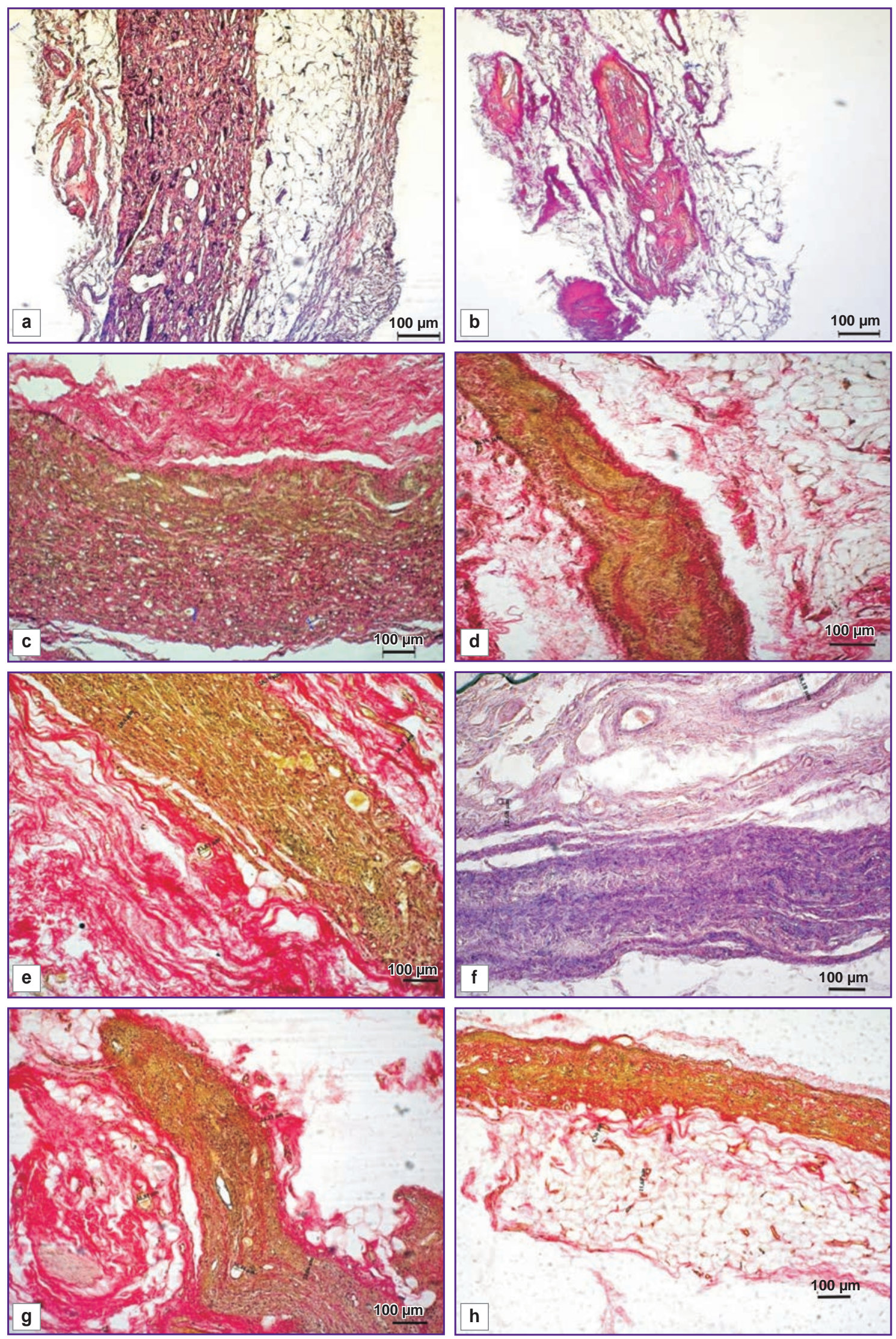

Figure 5. Nonwoven scaffolds 6 months postimplantation, $\times 100$ :

(a) PCL; (b) PHBV/PCL; (c) PCL+VEGF; (d) PHBV/PCL+VEGF; (e) PCL+bFGF; (f) PHBV/PCL+bFGF;

(g) PCL+SDF-1a; (h) PHBV/PCL+SDF-1a 

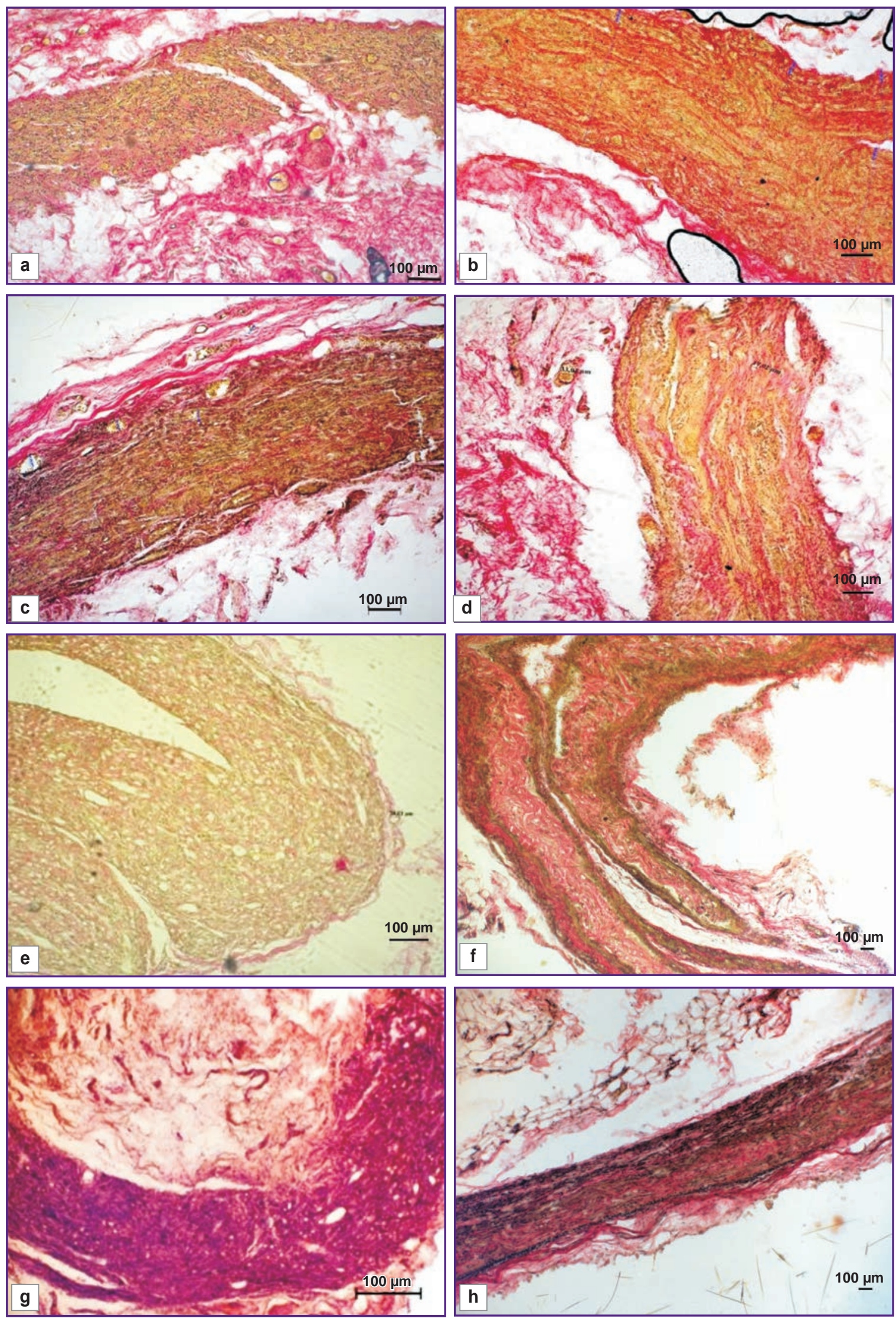

Figure 6. Nonwoven scaffolds 9 months postimplantation, $\times 100$ :

(a) PCL; (b) PHBV/PCL; (c) PCL+VEGF; (d) PHBV/PCL+VEGF; (e) PCL+bFGF; (f) PHBV/PCL+bFGF; (g) PCL+SDF-1a; (h) PHBV/PCL+SDF-1a 

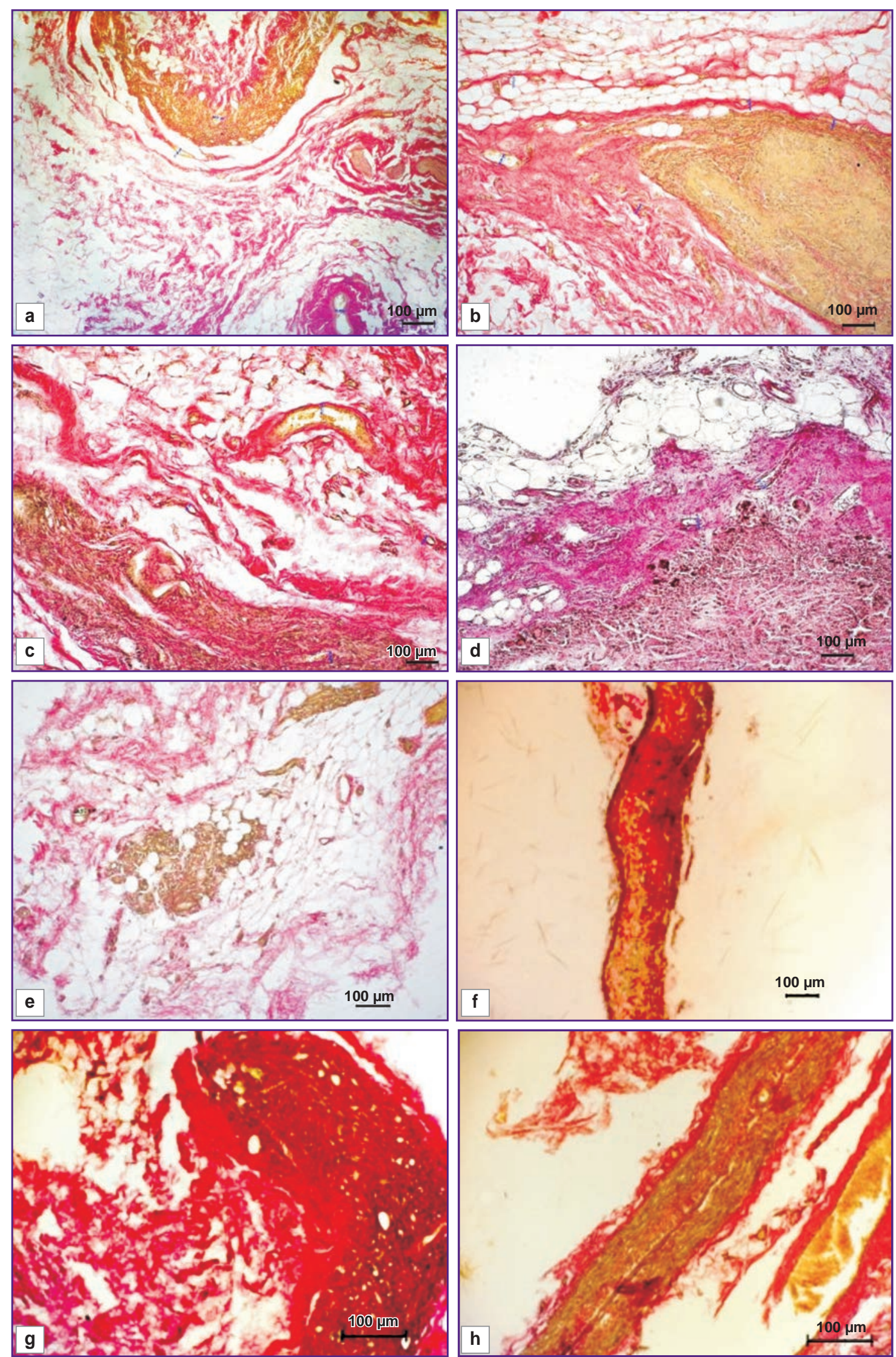

Figure 7. Nonwoven scaffolds 12 months postimplantation, $\times 100$ :

(a) PCL; (b) PHBV/PCL; (c) PCL+VEGF; (d) PHBV/PCL+VEGF; (e) PCL+bFGF; (f) PHBV/PCL+bFGF;

(g) PCL+SDF-1 $\alpha$; (h) PHBV/PCL+SDF-1 $\alpha$ 
In the PCL+SDF-1a group, there were no signs of inflammation (Figure $7(\mathrm{~g})$, (h)). Since the scaffold was degrading, gaps filled in with a loose connective tissue were formed.

Thus, incorporation of VEGF, bFGF and chemoattractant molecule SDF-1a into nonwoven scaffold during electrospinning did not result in their destruction during subcutaneous implantation, which was confirmed by their specific effects revealed on histological analysis. The most active angiogenesis (scaffolds with VEGF), formation of a thick connective tissue capsule (scaffolds with bFGF), enrichment of the cellular composition (scaffolds with SDF-1a) were registered in the first three months of subcutaneous implantation. Further on, some processes continued to develop. They were likely to be associated with the preservation of biological activity of these molecules as well as with the ongoing reactions, which were triggered in the first three months.

Conclusion. In subcutaneous implantation of nonmodified and biofunctionalized nonwoven scaffolds, aseptic inflammation has been observed. The most active angiogenesis and moderate cellular infiltration during the first three months are noted in the experimental specimens of the nonwoven scaffolds with incorporated VEGF. In the specimens with incorporated SDF-1a, active infiltration of the adjacent tissues by the cells synthesizing extracellular matrix is going on. Granulomatous inflammation in the perifocal zone started from the twelfth month of implantation reflects immune processes of the granulomatous inflammation type, signifying incomplete resorption of the nonwoven scaffolds.

Study Funding. The research was funded by Russian Science Foundation (project No.14-25-00050).

Conflicts of Interest. The authors have no conflicts of interest to disclose.

\section{References}

1. Catto V., Farè S., Freddi G., Tanzi M.C. Vascular tissue engineering: recent advances in small diameter blood vessel regeneration. ISRN Vascular Medicine 2014; 2014: 923030, https://doi.org/10.1155/2014/923030.

2. Hasan A. Memic A., Annabi N., Hossain M., Paul A., Dokmeci M.R., Dehghani F. Khademhosseini A. Electrospun scaffolds for tissue engineering of vascular grafts. Acta Biomater 2014; 10(1): 11-25, https://doi.org/10.1016/j. actbio. 2013.08.022.

3. Lee Y-S. Livingston Arinzeh T. Electrospun nanofibrous materials for neural tissue engineering. Polymers 2011; 3(4): 413-426, https://doi.org/10.3390/polym3010413.
4. Sankaran K.K., Subramanian A., Krishnan U.M., Sethuraman S. Nanoarchitecture of scaffolds and endothelial cells in engineering small diameter vascular grafts. Biotechnol J 2015; 10(1): 96-108, https://doi.org/10.1002/ biot.201400415.

5. Antonova L.V., Burago A.Y., Matveeva V.G., Kudryavtseva Y.A., Nasonova M.V., Toropova Y.G., Velikanova E.A., Golovkin A.S. Bioresorption characteristics of plain and cell-loaded polyhydroxyalkanoate and polycaprolactone film scaffolds in chronic long-term experiment. Fundamentalnie issledovania 2013; 7(1): 17-23.

6. Antonova L.V., Nasonova M.V., Kudryavtseva Yu.A., Golovkin A.S. Potential for polyhydroxyalkanoates and policaprolactone copolymer use as tissue-engineered scaffolds in cardiovascular surgery. Byulleten sibirskoy meditsiny 2012; 11(1): 128-134.

7. Popova I.V., Stepanova A.O., Plotnikova T.A., Sergeevichev D.S., Akulov A.E., Pokushalov A.A., Laktionov P.P., Karpenko A.A. Study of patency of vascular grafts manufactured by means of electrospinning. Angiologiya $i$ sosudistaya khirurgiya 2015; 21(2): 136-141.

8. de Valence S., Tille J.-C., Mugnai D., Mrowczynski W., Gurny R., Möller M., Walpoth B.H. Long term performance of polycaprolactone vascular grafts in a rat abdominal aorta replacement model. Biomaterials 2012; 33(1): 38-47, https:/ doi.org/10.1016/j.biomaterials.2011.09.024.

9. Rabkin E., Schoen F.J. Cardiovascular tissue engineering. Cardiovasc Pathol 2002; 11(6): 305-317, https:// doi.org/10.1016/s1054-8807(02)00130-8.

10. Finlay H.M., Whittaker P., Canham P.B. Collagen organization in the branching region of human brain arteries. Stroke 1998; 29(8): 1595-1601, https://doi.org/10.1161/01. str.29.8.1595

11. Natasha G., Tan A., Gundogan B., Farhatnia Y., Nayyer L., Mahdibeiraghdar S., Rajadas J., De Coppi P., Davies A.H., Seifalian A.M. Tissue engineering vascular grafts a fortiori: looking back and going forward. Expert Opin Biol Ther 2014; 15(2): 231-244, https://doi.org/10.1517/14712598. 2015.980234.

12. Azimi-Nezhad M. Vascular endothelial growth factor from embryonic status to cardiovascular pathology. Rep Biochem Mol Biol 2014; 2(2): 59-69.

13. Harburger D.S., Calderwood D.A. Integrin signalling at a glance. J Cell Sci 2009; 122(9): 1472-1472, https://doi. org/10.1242/jcs.052910.

14. GOST ISO 10993-1-2011. Medical devices. Biological evaluation of medical devices. Part 1. Evaluation and testing. 5.2.9 Implantation.

15. Nasonova M.V., Antonova I.V., Matveeva V.G., Doronina N.V., Ezhov V.A., Burago A.Y., Glushkova T.V., Kudryavtseva Yu.A. Resorption rate of polyhydroxyalkanoatebased scaffolds and scaffolds with multipotent mesenchymal stromal cells. Complex Issues of Cardiovascular Diseases 2015; 1: 39, https://doi. org/10.17802/2306-1278-2015-1-39-45. 